\title{
THE HUMAN OOCYTE-DERIVED SPERM CHEMOATTRACTANT IS A HYDROPHOBIC MOLECULE ASSOCIATED WITH A CARRIER PROTEIN
}

Leah Armon, Ph.D., ${ }^{a \dagger}$ Ido Ben-Ami, M.D., Ph.D., ${ }^{\mathrm{b}}$ Raphael Ron-El, M.D., ${ }^{\mathrm{b}}$ and Michael Eisenbach, Ph.D. ${ }^{a}$

${ }^{a}$ Department of Biological Chemistry, The Weizmann Institute of Science, 76100 Rehovot, Israel.

b IVF and Infertility Unit, Assaf Harofeh Medical Center, Sackler School of Medicine, Tel-Aviv University, 70300 Zerifin, Israel.

L.A. has nothing to disclose. I.B.-A. has nothing to disclose. R.R.-E. has nothing to disclose. M.E. has nothing to disclose.

Supported in part by a research grant from the Chief Scientist Office of the Ministry of Health, Israel, grant number 300000-5183.

Reprints requests: Michael Eisenbach, Ph.D., Department of Biological Chemistry, The Weizmann Institute of Science, 7610001 Rehovot, Israel (E-mail: m.eisenbach@weizmann.ac.il).

\footnotetext{
${ }^{\dagger}$ Current address: Kimron Veterinary Institute, 50250 Bet Dagan, Israel
} 


\section{Capsule}

Treating oocyte-conditioned media with proteases and hexane provided evidence for the oocyte-derived chemoattractant being a hydrophobic non-peptide molecule which, in an oocyteconditioned medium, is associated with a carrier protein. 


\begin{abstract}
Objective: To characterize the nature of the human oocyte-derived chemoattractant.

Design: Laboratory in-vitro study.

Setting: Academic research institute.

Patients: Ten healthy sperm donors. Oocyte-conditioned media from women undergoing IVF treatment due to male factor infertility.

Interventions: Sperm samples were processed by the migration-sedimentation technique. Oocyteconditioned media were collected 2-3 $\mathrm{h}$ after oocyte stripping.

Main Outcome Measure(s): Sperm chemotaxis was assayed in a $\mu$-slide chamber according to the direction of swimming relative to that of the chemical gradient.

Results: Oocyte-conditioned media treated with proteases did not lose their chemotactic activity; on the contrary, they became more active, with the activity shifted to lower concentrations. When oocyte-conditioned media were subjected to hexane extraction, chemotactic activity was found in both the hydrophobic and aqueous phases. Known mammalian sperm chemoattractants were ruled out as oocyte-derived chemoattractants.

Conclusions: Our results suggest that the oocyte-derived chemoattractant is a hydrophobic nonpeptide molecule which, in an oocyte-conditioned medium, is associated with a carrier protein that enables its presence in a hydrophilic environment.
\end{abstract}

\title{
Key words
}

Sperm chemotaxis; Chemotaxis; Oocyte-derived chemoattractant 


\section{Introduction}

In recent years it became clear that mammalian spermatozoa must be guided for reaching the oocyte [for a review, see (1)]. Thus far, three potential sperm guidance mechanisms have been recognized in humans: chemotaxis (2), thermotaxis (3), and rheotaxis (4). All these mechanisms only function in capacitated spermatozoa, i.e., ripe spermatozoa that acquired the ability to fertilize the egg (3-5). Thermotaxis and rheotaxis are assumed to be long-range mechanisms (1,4) guiding spermatozoa to the fertilization site, and chemotaxis is thought to be a short-range, more accurate mechanism that acts in the immediate surroundings of the oocyte-cumulus complex, guiding spermatozoa to the oocyte (1). This short-range mechanism appears to be a two-step process, first chemotaxis to the cumulus cells that surround the oocyte and then chemotaxis within the cumulus mass towards the oocyte itself. This is based on the finding that, in humans, there are two origins of sperm chemoattractants: the mature oocyte and the surrounding cumulus cells (6). The cumulus cells secrete a single chemoattractant, progesterone $(7,8)$. This chemoattractant is highly active, with chemoattraction being observed at a concentration range as low as $10^{-11}-10^{-10} M(7,8)$. The identity of the chemoattractant(s) secreted from the oocyte is not known, in spite of intensive efforts to identify it. It is known, however, that it is more potent than the cumulus-derived chemoattractant progesterone (6), meaning that it is probably active at concentrations lower than $10^{-11} \mathrm{M}$. Realizing that the identification of a substance present at such a low concentration is extremely difficult, our aim in this study was to characterize the nature of the oocyte-derived chemoattractant.

\section{Materials and Methods}

\section{Chemicals}

Modified human tubal fluid (HTF) medium and human serum albumin (HSA) solution were obtained from Irvine Scientific (Santa Ana, CA, USA); polyvinylpyrrolidone (PVP, 25K) was from Fluka 
(Buchs, Switzerland); progesterone, Pronase E and undecanal were purchased from Sigma (Rehovot, Israel); Proteinase K and $n$-hexane were from Merck (Darmstadt, Germany) and prostaglandin E1 was purchased from Avanti Polar Lipids (AL, USA).

\section{Spermatozoa}

Human semen samples were obtained from ten healthy donors after 3 days of sexual abstinence. (The study was approved by the Bioethics and Embryonic Stem Cell Research Oversight Committee of the Weizmann Institute of Science. Informed consent was obtained in writing from each sperm donor.) Semen samples were allowed to liquefy for 30-60 min at room temperature and verified for normal sperm density, motility and morphology [according to WHO guidelines (9)]. Human spermatozoa were separated from the seminal plasma by the migration-sedimentation technique (10) using the commercially available HTF medium supplemented with $0.3 \%(\mathrm{w} / \mathrm{v}) \mathrm{HSA}$. Following this procedure, the sperm concentration was adjusted to $4-5 \times 10^{5}$ cells $/ \mathrm{ml}$ in HTF medium containing $0.3 \% \mathrm{HSA}$ and $3.5 \%(\mathrm{w} / \mathrm{v})$ PVP. The sperm suspensions were incubated under an atmosphere of $5 \%(\mathrm{v} / \mathrm{v}) \mathrm{CO}_{2}$ at $37^{\circ} \mathrm{C}$ for an additional $1 \mathrm{~h}(2 \mathrm{~h}$ in total with the separation procedure) to obtain capacitated spermatozoa (5).

\section{Oocyte-conditioned media}

Oocyte-conditioned media and the respective control medium (G-1, Vitrolife, Sweden) were obtained from the In-vitro Fertilization (IVF) Unit at Assaf Harofeh Medical Center (with the approvals of the hospital's Helsinki committee and the Weizmann Institute Bioethics and Embryonic Stem Cell Research Oversight Committee). Cumulus-oocyte complexes were retrieved from women undergoing transvaginal oocyte aspiration for in vitro fertilization. The women were treated with GnRH analog and gonadotrophin for ovarian stimulation. Oocyte retrieval was performed $35-38 \mathrm{~h}$ after human choriogonadotrophin injection. The cumulus cells were stripped from the cumulus-oocyte complexes by employing both mechanical pipetting and $10-30 \mathrm{~s}$ treatment with 40 units/mg hyaluronidase 
(type IV-S, Sigma, USA) followed by washing, thus avoiding leftovers of cumulus cells. Groups of stripped oocytes (6-8 per group) were cultured in $25 \mu$ drops of G-1 medium supplemented with $10 \%(\mathrm{v} / \mathrm{v}) \mathrm{HSA}$ for 2-3 $\mathrm{h}$ before undergoing intra-cytoplasmic sperm injection. The conditioned media were collected and frozen just prior to the injection.

\section{Progesterone determination}

Oocyte-conditioned media were analyzed for the presence of progesterone using a progesterone ELISA kit (Enzo Life Sciences, Farmingdale, NY, USA) according to manufacturer instructions. The concentration of progesterone was calculated according to an experimental standard curve. The lowest concentration of the standard was $15.62 \mathrm{pg} / \mathrm{ml}$, which set up the lowest limit of detection. Media from six different women pooled in pairs (total of three measured samples) were subjected to analysis. Samples were diluted 1:14 and run in duplicates. The negative control was G-1 medium supplemented with $10 \%(\mathrm{v} / \mathrm{v}) \mathrm{HSA}$.

\section{Hexane extraction}

An equal volume of $n$-hexane was added to the samples of oocyte-conditioned medium and control medium. The tubes were vortexed for $2 \mathrm{~min}$ and then spun $(3000 \times \mathrm{g})$ until a clear differentiation between two phases was observed. The upper phase, containing the hexane fraction, was transferred to another tube. Both fractions were then subjected to chemotaxis assays.

\section{Chemotaxis assays}

Chemotaxis assays were performed as described (11), using a disposable $\mu$-slide chemotaxis chamber (Ibidi GmBH, Munich, Germany). Each assay included a negative control experiment in the absence of the test substance. In experiments with oocyte-conditioned media this negative control consisted of a gradient of the oocyte-incubating medium [G-1 medium supplemented with $10 \%(\mathrm{v} / \mathrm{v})$ HSA] treated the same way as the oocyte-conditioned medium (e.g., protease treatment). In experiments with prostaglandin E1 the negative control contained a gradient of the diluent. 


\section{Chemotaxis assessment and statistics}

Chemotaxis was assessed, as described (12), according to the distribution of the instantaneous directionality angles $\left(\gamma_{\text {inst }}\right.$, the angle between the vector of the cell frame-to-frame displacement and the gradient direction; the video frequency was 25 frames/s). For each treatment the total number of angles in the gradient direction $(\mathrm{N}+)$ and in the opposite direction $(\mathrm{N}-)$ were summed from a number of experiments. A combined odds parameter was calculated as the ratio between these sums (combined Odds $=\Sigma \mathrm{N}+/ \Sigma \mathrm{N}-$ ). The odds parameter yields values close to 1 when the swimming is random; it is $>1$ when the swimming is biased in the gradient direction. The intensity of the chemotactic response was reflected in the combined odds ratio (O.R.) parameter $\left(\right.$ combined O.R. $=$ combined Odds $_{\text {treatment }} /$ combined Odds $\left._{\text {control }}\right)$.

To assess the statistical significance of elevated O.R. we used a block-bootstrapping algorithm as described earlier (13). Briefly, we resampled a large control data set to build a distribution of control O.R. for different sample sizes. The distribution provided the probability that a treatment O.R. is above a control O.R. randomly drawn from this distribution. The assay variability was assessed by the width of the control confidence intervals, assuming similar variability for the treatments and the controls. The dependence of the confidence intervals of the control O.R. values on the sample size is shown in Table 1.

\section{Results}

Wishing to employ for the characterization the most physiologically relevant source of oocyte-derived chemoattractant, we used human oocyte-conditioned media, i.e., media in which mature oocytes, stripped from the surrounding cumulus cells, were incubated for $2-3 \mathrm{~h}$. We assayed chemotaxis by determining the swimming bias in the direction of the chemoattractant gradient, and calculating the ratio between the biases in the presence and absence of a gradient (termed combined odds ratio - see Materials and Methods). A value of 1 means no chemotaxis; a value $>1$ 
means chemotaxis towards the chemoattractant $(12,13)$. Fig. $1 \mathrm{~A}$ shows a representative chemotactic response of oocyte-conditioned media at various dilutions, demonstrating that an oocyteconditioned medium is usually chemotactically active at $10^{5}-10^{6}$ dilution.

To verify that these conditioned media had no remains of progesterone from the cumulus cells, we performed a highly sensitive, progesterone-specific ELISA assay. We assayed three different samples of oocyte-conditioned media, each being a mixture of oocyte-conditioned media obtained from two women (6-8 oocytes each). In two of the samples, no progesterone was detected by the assay. In the third sample, progesterone at a concentration of $2 \times 10^{-9} \mathrm{M}$ was detected. Since oocyteconditioned media are chemotactically active at $10^{5}-10^{6}$ dilutions (Fig. $1 \mathrm{~A}$ ), the effective concentration of progesterone in the diluted sample was in the range of $2 \times\left(10^{-15}-10^{-14}\right) \mathrm{M}$, which is four orders of magnitude below the threshold progesterone concentration that can be sensed by human spermatozoa and stimulate a chemotactic response $\left[10^{-11}-10^{-10} \mathrm{M}(7,8)\right]$.

To characterize the nature of the oocyte-derived chemoattractant, we first determined whether or not it is a peptide. We incubated oocyte-conditioned media with a broad-spectrum protease, Proteinase K. In each experiment we verified the activity of the protease by running the oocyte-conditioned media on SDS-PAGE prior and subsequent to the proteolysis. The chemoattractant activity of the oocyte-conditioned media was not lost by the proteolysis (Fig. 1B; similar results were obtained with trypsin - data not shown). On the contrary, the activity shifted to a lower concentration (i.e., higher dilution). Since Proteinase K cleaves peptide bonds adjacent to the carboxyl group of both aliphatic and aromatic amino acids, these results suggest that the chemoattractant in oocyte-conditioned media is not a peptide (excluding a very short peptide).

To verify the unexpected observation that the proteolysis increased the chemotactic activity of the medium from $1: 10^{5}$ dilution to $1: 10^{6}$, we carried out another set of experiments, using this time a mixture of two proteases, Proteinase $\mathrm{K}$ and Pronase $\mathrm{E}$ in higher concentrations than those 
used for Proteinase $\mathrm{K}$ alone, thus eliminating any possibility of peptide leftovers (excluding very small peptides). In this set of experiments the sample of oocyte-conditioned media (prior to treatment) happened to be chemotactically inactive. (This happened occasionally due to the fact that we used a pool of media from different women.) Nevertheless, these inactive media became active after proteolysis (Fig. 1C). Clearly, proteolysis elevated the chemoattractant concentration. This could be the outcome of two scenarios. One is that the chemoattractant is an amino acid or a very short peptide (such as a di-or tri-peptide) and proteolysis produces more of it. The other is that the chemoattractant is a hydrophobic molecule which, in an oocyte-conditioned medium, is associated with a carrier protein that enables its presence in a hydrophilic environment. According to this scenario, proteolysis destroys the carrier protein and frees the hydrophobic chemoattractant to the medium (the high concentration of albumin in the medium likely enables presence of the chemoattractant in the aqueous solution).

If, indeed, the oocyte-derived chemoattractant is hydrophobic and it is chemotactically active both when it is in complex with its carrier protein and when unbound, it should exhibit chemotactic activity both in hydrophilic and hydrophobic environments. To determine whether or not this is the case, we extracted by hexane the hydrophobic substances from the oocyteconditioned media, and examined the chemotactic activity of both the hexane fraction and the remaining aqueous fraction. Consistently with the carrier-protein scenario, both fractions were chemotactically active (Fig. 2).

Two sperm receptors apparently can act as chemotaxis receptors. One of them is the CatSper channel (or a protein closely associated with it), shown to be the sperm receptor for the chemoattractant progesterone $(14,15)$. The other is OR1D2, suggested to mediate the chemotactic response to the odorant bourgeonal (16) [though a later study raised the possibility that the response to bourgeonal is mediated by CatSper (17)]. Since the ligands of these receptors are quite hydrophobic, we examined whether any of them is the chemoattractant in oocyte-conditioned 
medium. CatSper is also activated by prostaglandin E1 (though via a binding site different from that of progesterone) $(14,15)$ and is known to be secreted from the cumulus-oocyte complex $(18)$. Prostaglandin E1 exhibited no chemotactic activity at any tested concentration (Fig. 3A), even though it bound to its receptor (reflected in stimulating intracellular $\mathrm{Ca}^{2+}$ rise - not shown). Likewise, preincubation of oocyte-conditioned media with undecanal - an antagonist of the OR1D2 receptor (16), did not affect the chemotactic activity of the medium (Fig. 3B). These results indicate that neither prostaglandin E1 nor an OR1D2 ligand is the oocyte-derived chemoattractant.

\section{Discussion}

The results of this study show that the human oocyte-derived sperm chemoattractant does not lose its activity by proteases (Fig. 1), it can be present in both hydrophobic and hydrophilic environments (Fig. 2), and it is not one of the known ligands of the recognized chemotaxis receptors (Fig. 3). The first two observations could be explained by a number of scenarios: a hydrophobic chemoattractant in complex with a carrier protein, two (or more) chemoattractants - one hydrophobic and one hydrophilic, a chemoattractant of an amphipathic nature, and a chemoattractant which is an amino acid or a very short peptide. The finding that the activity of the oocyte-conditioned medium increased by proteolysis, taken together with the conservation of activity in both hydrophobic and hydrophilic environments, is consistent with the possibility of a hydrophobic chemoattractant associated with a carrier protein in an aqueous environment. According to this possibility, both the hydrophobic molecule and its complex with the carrier protein are chemotactically active. The free hydrophobic molecule is expected to be more active because, otherwise, proteolysis would not increase the activity. The other mentioned scenarios do not seem probable. Thus, the possibility of an amphipathic chemoattractant or two chemoattractants, one hydrophobic and one hydrophilic, is inconsistent with the proteolysis-dependent increase in chemotactic activity. Likewise, the possibility of an amino acid chemoattractant or a very short 
chemoattractant peptide is inconsistent with the proteolysis results by trypsin, which generates relatively long peptides. The anticipation in such a case would be no change in activity. The results further indicate that the oocyte-derived chemoattractant is not one of the peptides reported to act in vitro as chemoattractants for human spermatozoa [e.g., CCL5 (19), Allurin (20), CCL20 (21) and atrial natriuretic peptide (22)] as all are expected to undergo proteolysis by the proteases used. Thus, it seems probable that the chemoattractant is a hydrophobic molecule associated with a carrier protein.

It is reasonable to speculate that the receptor for the oocyte-derived chemoattractant is different from the known receptors for sperm chemoattractants. Otherwise, the receptor for the oocyte-derived chemoattractant might be saturated with cumulus-derived progesterone when passing through the mass of cumulus cells, thus interfering with the binding of the oocyte-derived chemoattractant to this receptor.

To conclude, the chemoattractant is likely a hydrophobic non-peptide molecule which, when secreted from the oocyte, is in complex with a carrier protein. Apparently, the extreme potency of the chemoattractant compensates for its low concentration in the medium, and the carrier protein enables the presence of the chemoattractant in aqueous solutions and prevents its adsorption to non-specific locations.

\section{Acknowledgments}

We thank to Dr. Milana Fraiberg and Dr. Serafin Pérez-Cerezales for so willingly performing the ELISA assays for us. We are also grateful to the embryologists team at Assaf Harofeh Medical Center for collecting the oocyte-conditioned media. M.E. is an incumbent of the Jack and Simon Djanogly Professorial Chair in Biochemistry. 


\section{References}

1. Eisenbach M, Giojalas LC. Sperm guidance in mammals - an unpaved road to the egg. Nature Rev Mol Cell Biol 2006;7(4):276-85.

2. Ralt D, Manor M, Cohen-Dayag A, Tur-Kaspa I, Makler A, Yuli I, et al. Chemotaxis and chemokinesis of human spermatozoa to follicular factors. Biol Reprod 1994;50:774-85.

3. Bahat A, Tur-Kaspa I, Gakamsky A, Giojalas LC, Breitbart H, Eisenbach M. Thermotaxis of mammalian sperm cells: A potential navigation mechanism in the female genital tract. Nat Med 2003;9(2):149-50.

4. Miki K, Clapham DE. Rheotaxis Guides Mammalian Sperm. Curr Biol 2013;23:443-52.

5. Cohen-Dayag A, Tur-Kaspa I, Dor J, Mashiach S, Eisenbach M. Sperm capacitation in humans is transient and correlates with chemotactic responsiveness to follicular factors. Proc Natl Acad Sci USA 1995;92(24):11039-43.

6. Sun F, Bahat A, Gakamsky A, Girsh E, Katz N, Giojalas LC, et al. Human sperm chemotaxis: both the oocyte and its surrounding cumulus cells secrete sperm chemoattractants. Hum Reprod 2005;20(3):761-7.

7. Teves ME, Barbano F, Guidobaldi HA, Sanchez R, Miska W, Giojalas LC. Progesterone at the picomolar range is a chemoattractant for mammalian spermatozoa. Fertil Steril 2006;86(3):745-9.

8. Oren-Benaroya R, Orvieto R, Gakamsky A, Pinchasov M, Eisenbach $M$. The sperm chemoattractant secreted from human cumulus cells is progesterone. Hum Reprod 2008;23:2339-45.

9. World Health Organization. WHO Laboratory manual for the examination and processing of 
human semen. 5 ed. Geneva, Switzerland: WHO Press; 2010.

10. Hauser R, Homonnai ZT, Paz GF, Yavetz H, Amit A, Lessing JB, et al. Migration sedimentation technique as a predictive test for the fertilizing capacity of spermatozoa in anin vitrofertilization programme. Int J Androl 1992;15(6):498-503.

11. Armon L, Eisenbach M. Behavioral mechanism during human sperm chemotaxis: Involvement of hyperactivation. PLoS ONE 2011;6(12):e28359.

12. Gakamsky A, Schechtman E, Caplan SR, Eisenbach M. Analysis of chemotaxis when the fraction of responsive cells is small - application to mammalian sperm guidance. Int J Dev Biol 2008;52(5-6):481-7.

13. Armon L, Caplan SR, Eisenbach M, Friedrich BM. Testing human sperm chemotaxis: how to detect biased motion in population assays. PLoS ONE 2012;7(3):e32909.

14. Strunker T, Goodwin N, Brenker C, Kashikar ND, Weyand I, Seifert R, et al. The CatSper channel mediates progesterone-induced Ca2+influx in human sperm. Nature 2011;471(7338):382-6.

15. Lishko PV, Botchkina IL, Kirichok Y. Progesterone activates the principal Ca2+channel of human sperm. Nature 2011;471(7338):387-91.

16. Spehr M, Gisselmann G, Poplawski A, Riffell JA, Wetzel CH, Zimmer RK, et al. Identification of a testicular odorant receptor mediating human sperm chemotaxis. Science 2003;299(5615):2054-8.

17. Brenker C, Goodwin N, Weyand I, Kashikar ND, Naruse M, Krahling M, et al. The CatSper channel: a polymodal chemosensor in human sperm. EMBO J 2012;31(7):1654-65.

18. Viggiano JM, Herrero MB, Cebral E, Boquet MG, de Gimeno MF. Prostaglandin synthesis by 
cumulus-oocyte complexes: effects on in vitro fertilization in mice. Prostaglandins Leukotrienes and Essential Fatty Acids 1995;53(4):261-5.

19. Isobe T, Minoura H, Tanaka K, Shibahara T, Hayashi N, Toyoda N. The effect of RANTES on human sperm chemotaxis. Hum Reprod 2002;17(6):1441-6.

20. Burnett LA, Anderson DM, Rawls A, Bieber AL, Chandler DE. Mouse sperm exhibit chemotaxis to allurin, a truncated member of the cysteine-rich secretory protein family. Dev Biol 2011;360(2):318-28.

21. Caballero-Campo P, Buffone MG, Benencia F, Conejo-García JR, Rinaudo PF, Gerton GL. A role for the chemokine receptor CCR6 in mammalian sperm motility and chemotaxis. J Cell Physiol 2014;229(1):68-78.

22. Zamir N, Riven-Kreitman R, Manor M, Makler A, Blumberg S, Ralt D, et al. Atrial natriuretic peptide attracts human spermatozoain vitro. Biochem Biophys Res Commun 1993;197(1):116-22.

23. Heuchel V, Schwartz D, Czyglik F. Between and within subject correlations and variances for certain semen characteristics in fertile men. Andrologia 1983;15(2):171-6.

24. Keel BA. Within- and between-subject variation in semen parameters in infertile men and normal semen donors. Fertil Steril 2006;85(1):128-34.

25. Gakamsky A, Armon L, Eisenbach M. Behavioral response of human spermatozoa to a concentration jump of chemoattractants or intracellular cyclic nucleotides. Hum Reprod 2009;24(5):1152-63. 


\section{Figure legends}

Fig. 1. Chemotactic response of human spermatozoa to oocyte-conditioned medium (OCM) in the absence and presence of proteases. A: Dependence of the chemotactic response on the OCM dilution. B: Chemotactic activity of OCM following treatment with Proteinase $\mathrm{K}\left(20 \mu \mathrm{g} / \mathrm{ml}, 3 \mathrm{~h}, 37^{\circ} \mathrm{C}\right)$. C: Chemotactic activity of OCM following treatment with a mixture of Proteinase K and Pronase E (50 $\mu \mathrm{g} / \mathrm{ml}$ each, $3 \mathrm{~h}, 37^{\circ} \mathrm{C}$ ). The data are presented as combined odds ratios (O.R.) of 6900-9500 tracks [(91-121) $\times 10^{4}$ angles; A] and 5000-5800 tracks $\left[(70-80) \times 10^{4}\right.$ angles; B,C]. The negative control is defined as O.R. $=1$. Note that error bars are missing because the combined O.R. value is calculated from a pool of the whole data and is not an average. Also note that, due to the large variations between sperm samples [even of the very same sperm donor - a well known phenomenon in sperm biology (23-25)], a quantitative comparison is allowed within each panel but not between panels. ${ }^{*} P$ $<0.05$ versus the appropriate control.

Fig. 2. Chemotactic response of human spermatozoa to oocyte-conditioned medium (OCM) before and after extraction with hexane. The data are presented as combined O.R. of 1400-2900 tracks $\left[(18-35) \times 10^{4}\right.$ angles]. Note that error bars are missing because the combined O.R. value is calculated from a pool of the whole data and is not an average. ${ }^{*} P \leq 0.06$; ${ }^{* *} P<0.01$ versus the appropriate control.

Fig. 3. Examination of sperm receptors suspected to be involved in human sperm chemotaxis. A: Chemotactic response to Prostaglandin E1 (PGE1). The data are presented as combined O.R. of 4200-

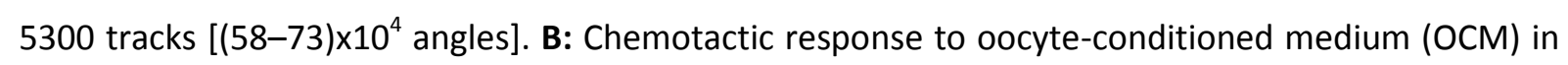
the presence of undecanal. The data are presented as combined O.R. of 2500-3600 tracks [(915) $\times 10^{4}$ angles]. Note that error bars are missing because the combined O.R. value is calculated from a pool of the whole data and is not an average. ${ }^{*} P \leq 0.02$ versus the appropriate control. 
Table 1

Confidence intervals (95\%) of O.R. values according to sample size

\begin{tabular}{cc}
\hline Sample size (number of angles) & Confidence interval $^{\mathrm{a}}$ \\
\hline $5 \times 10^{4}$ & $0.86-1.16$ \\
$10 \times 10^{4}$ & $0.90-1.11$ \\
$100 \times 10^{4}$ & $0.97-1.03$ \\
\hline
\end{tabular}

${ }^{\text {a }}$ Calculated by block-bootstrapping 


\section{Figure 1}

Click here to download high resolution image
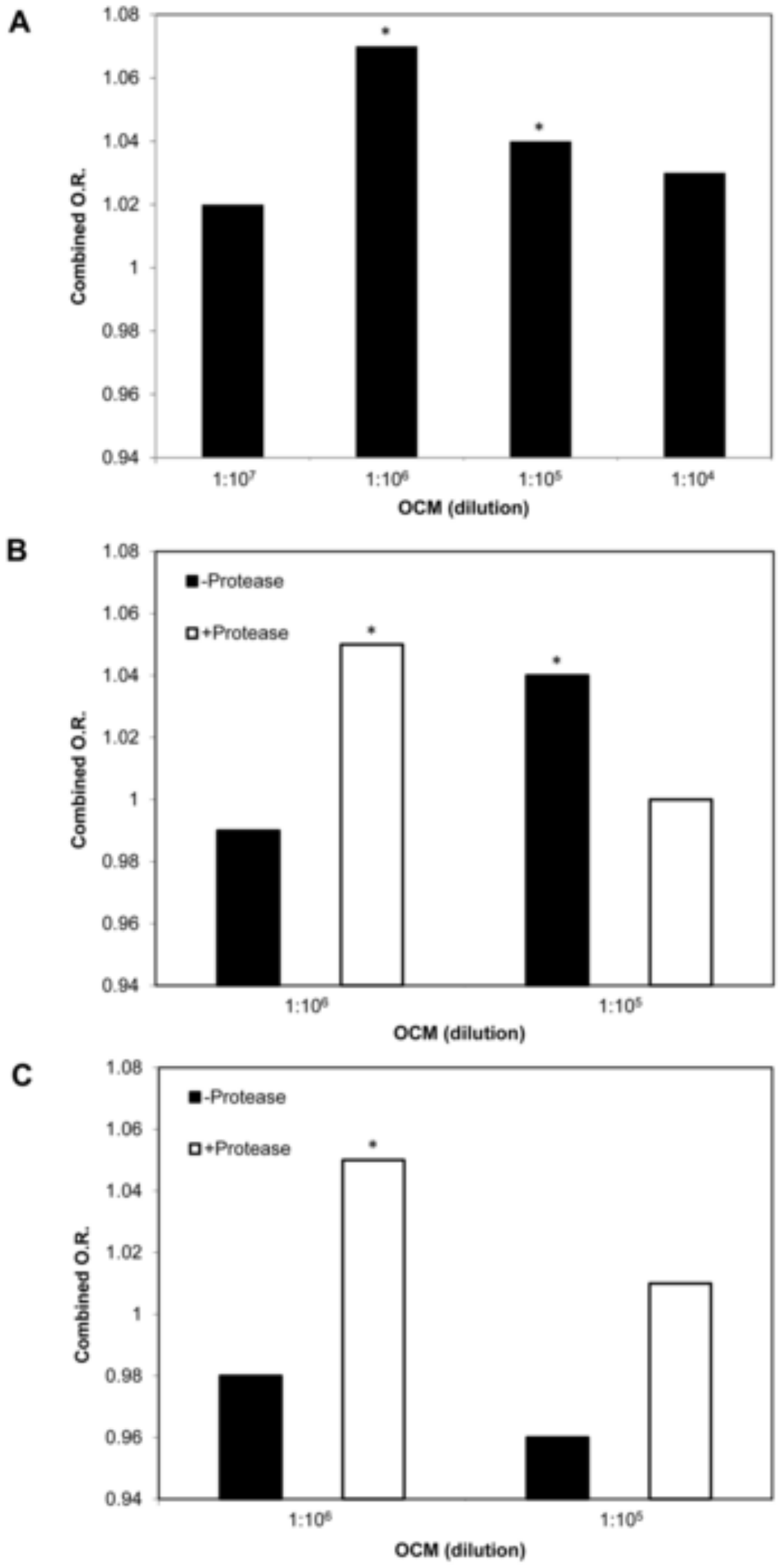
Click here to download high resolution image

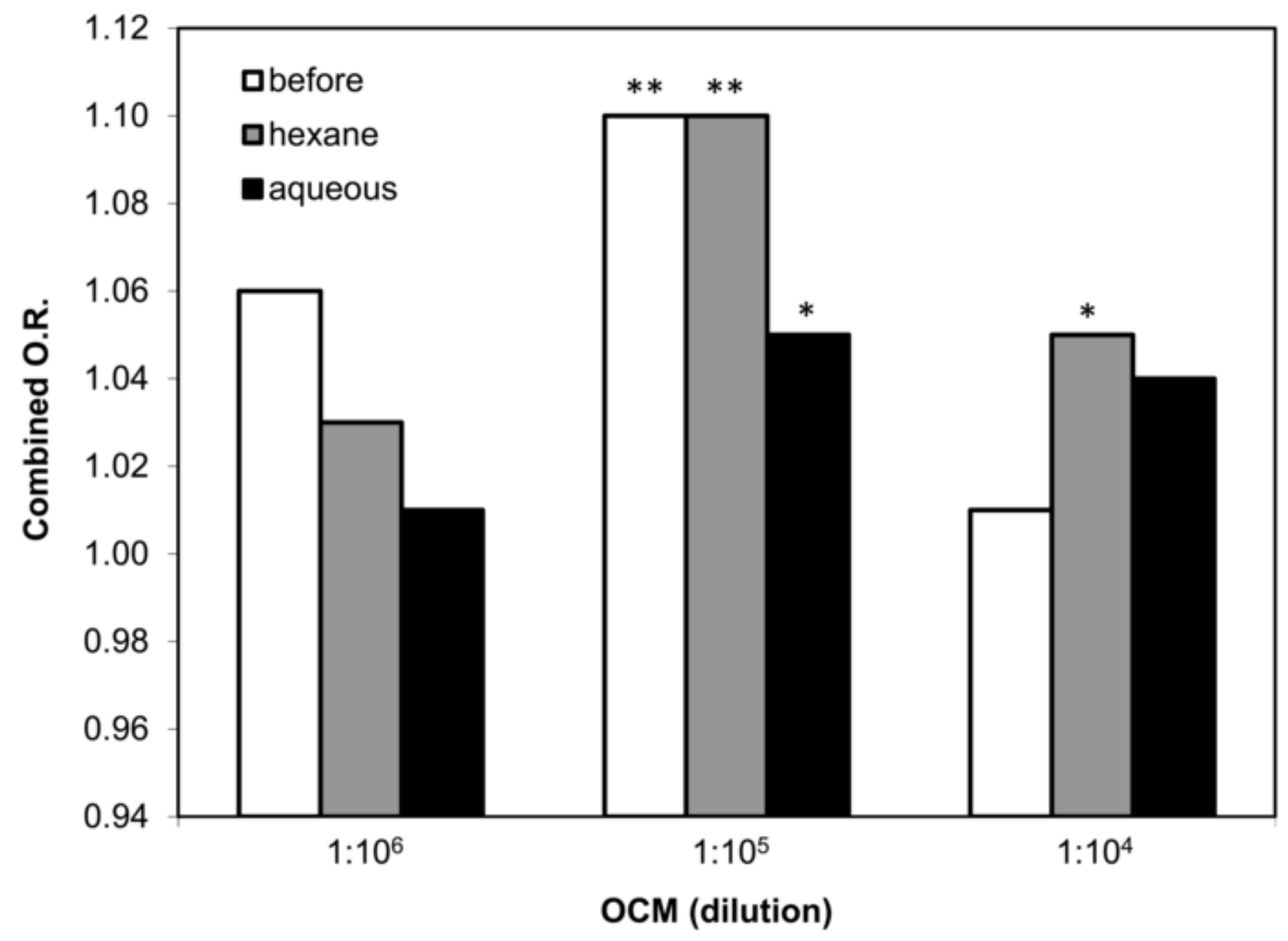

Click here to download high resolution image 
Figure 3

Click here to download high resolution image

A

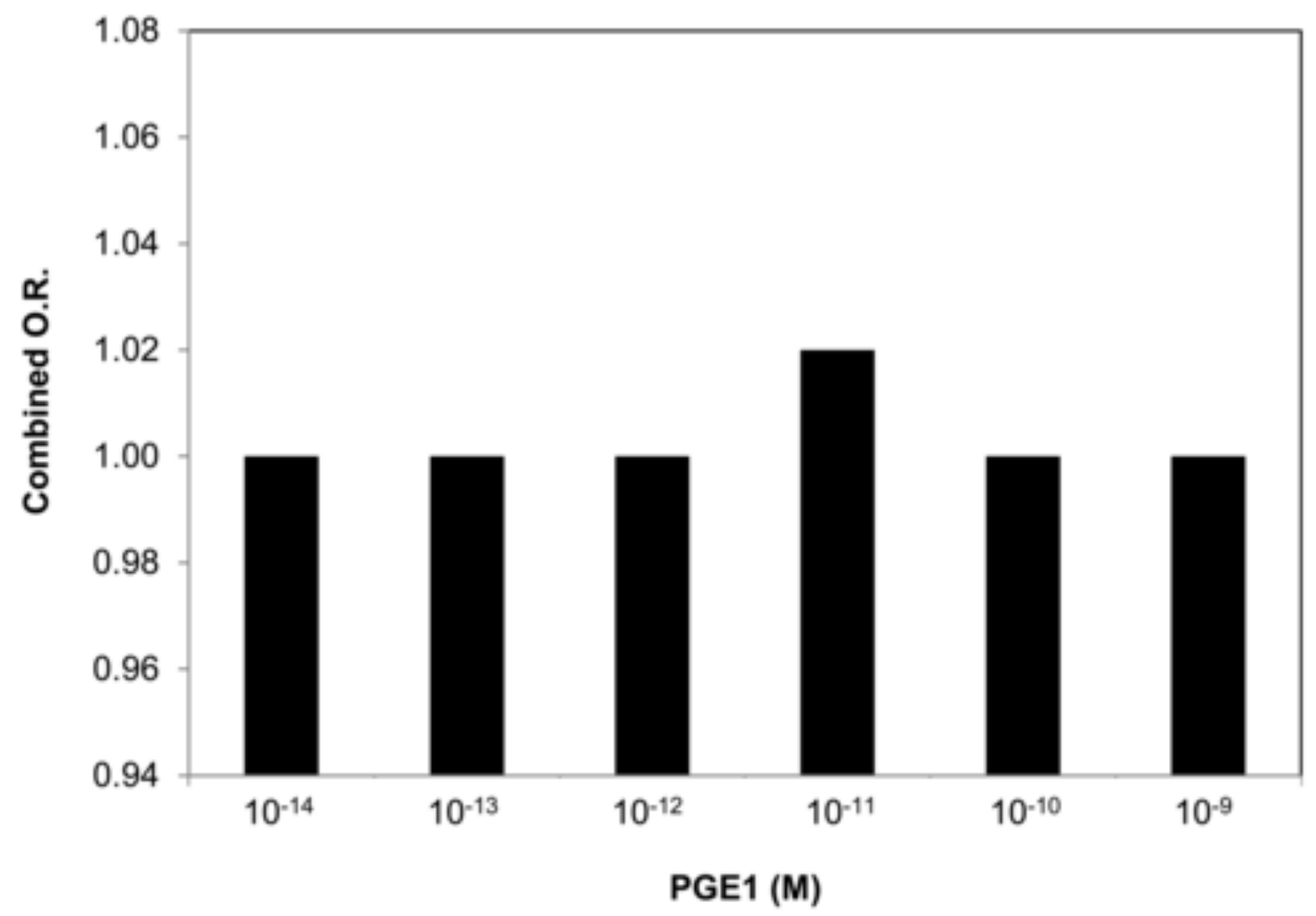

B

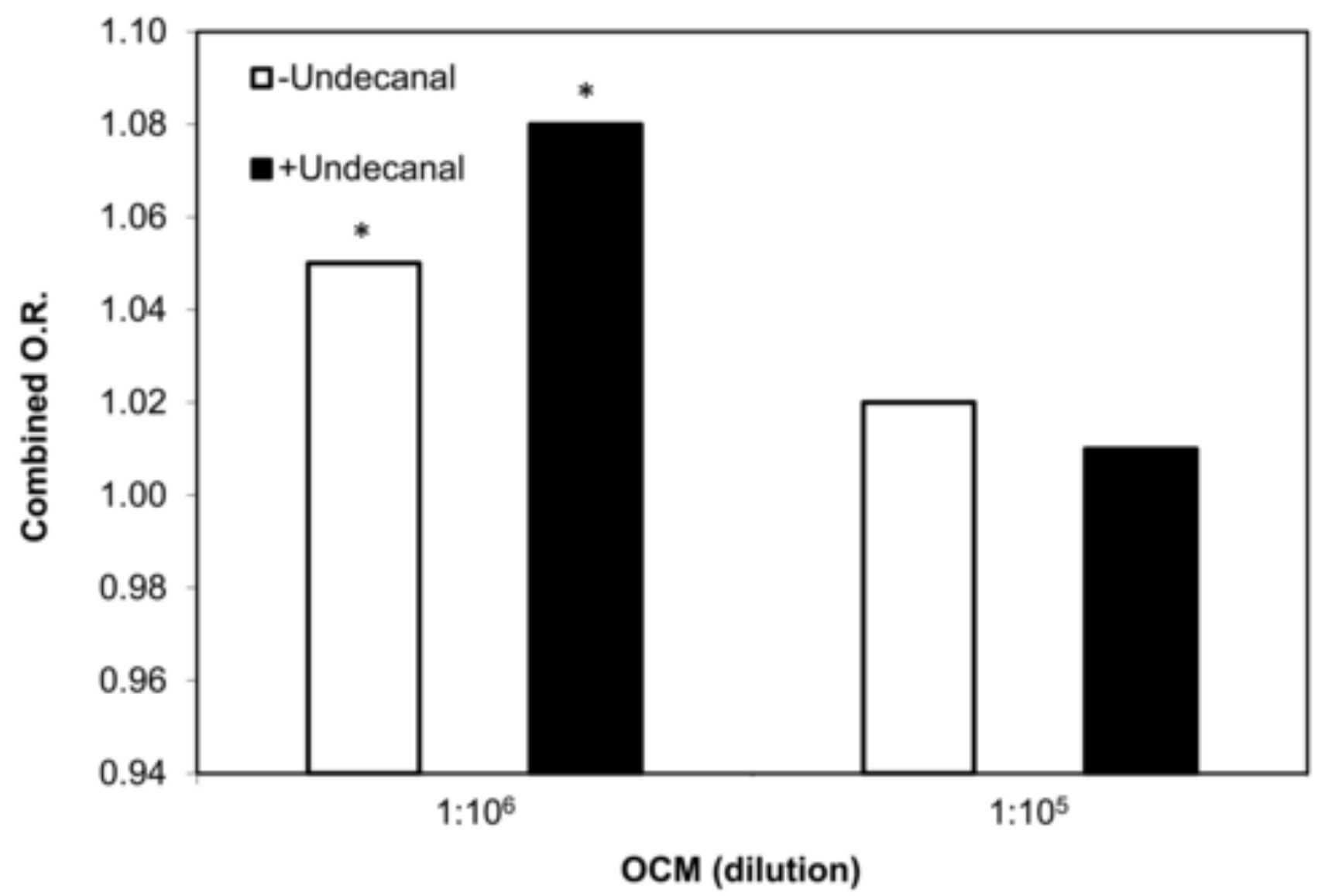

